\title{
Polymerbasierter Nachweis von Silber-Nanopartikeln mit Hilfe eines optischen und markierungsfreien Sensors
}

\author{
J.Adam ${ }^{1}$, J.Widmaier ${ }^{1}$ und G. Gauglitz ${ }^{1}$ \\ ${ }^{1}$ Institut für physikalische und theoretische Chemie, Eberhard Karls Universität Tübingen, Tübingen, \\ Deutschland \\ Kontakt: Jennifer.Adam@student.uni-tuebingen.de
}

\begin{abstract}
Zusammenfassung:
Nanopartikel kommen in vielen Produkten des täglichen Lebens vor. Deshalb ist es wichtig Nachweismethoden zu entwickeln, mit deren Hilfe Nanopartikel in unterschiedlichsten Matrices qualitativ und quantitativ nachgewiesen werden können. Nur so können auch Aussagen über ein mögliches Risikopotential von Nanopartikeln getroffen werden. Der Nachweis von Nanopartikeln ist jedoch nach wie vor eine herausfordernde Aufgabe, da nicht nur die Extraktion aus komplexen Matrices ein Problem darstellt, sondern sie sich auch in ihrer Größe und ihrem Material unterscheiden. Mit Hilfe der Reflektometrischen Interferenzspektroskopie (RIfS), die als optische und markierungsfreie Methode zum Nachweis verschiedener Analyten eingesetzt werden kann, wurde ein Versuch unternommen, Silber-Nanopartikel in wässriger Lösung qualitativ nachzuweisen. Die RIfS ermöglicht die zeitaufgelöste Verfolgung von Bindungsprozessen, wobei Bindungssignale aus Wechselwirkungen zwischen einem Analyten und einer sensitiven Schicht resultieren. Zum Nachweis der Partikel wurde eine, auf einem Polycinnamat basierende, sensitive Schicht eingesetzt, mit der Silber-Nanopartikel unterschiedlicher Größe erfolgreich detektiert werden konnten.
\end{abstract}

Schlagwörter: Reflektometrische Interferenzspektroskopie (RIfS), Sensor, Silber-Nanopartikel, Polymer, Polycinnamat

\section{Einleitung}

Die Bedeutung der Nanotechnologie wächst zunehmend, so dass technisch hergestellte Nanopartikel schon in vielen Alltagsprodukten zu finden sind. Aufgrund ihrer vielfältigen Eigenschaften bieten Nanopartikel (NPs) ein breites Anwendungsspektrum so z.B. in Kosmetika, Lebensmitteln oder Arzneimitteln. Titandioxid, eins der wohl bekanntesten Beispiele, ist z.B. in Sonnenschutzmitteln enthalten [1]. Silber-Partikel hingegen werden aufgrund ihrer antimikrobiotischen Eigenschaften z.B. in Wundverbänden eingesetzt [2].

Dem offensichtlichen Nutzen von Nanopartikeln stehen jedoch offene Fragen z.B. in Bezug auf die Risiken für Mensch und Umwelt gegenüber. So stehen z.B. Titandioxid-Partikel im Verdacht, Krebs auszulösen [3]. Dadurch werden auch Konsumenten zunehmend verunsichert. 2008 und 2012 wurden Konsumentenstudien zum Thema "Nanotechnologien aus der Sicht der Konsumenten" durchgeführt [4]. Vergleicht man die Ergebnisse der beiden Studien miteinander, so zeigt sich, dass die Zahl der Teilnehmer, die dem Einsatz von Nanopartikeln positiv gegenüberstehen, im Laufe der Zeit um 22\% abgenommen hat (2008: 64\%, 2012: 42\%) [4].

Gerade im Hinblick auf mögliche Risiken, die von NPs ausgehen, ist es wichtig, eine effiziente Analysemethode zu entwickeln, die z.B. in der Nahrungsmittelüberwachung oder Umweltanalytik eingesetzt werden kann, um NPs nachzuweisen. Ein Problem hierbei ist jedoch die Extraktion von NPs aus komplexen Matrices wie z.B. Cremes, die jedoch nötig ist, da Matrixkomponenten oft einen Effekt auf die Messergebnisse haben. Hierzu soll im Rahmen des Projekts INSTANT (Innovative Sensor for the Analysis of Nanoparticles in Selected Target Products) ein kostengünstiges Gerät entwickelt werden, dass einen qualitativen und quantitativen Nachweis verschiedener Nanopartikel ermöglicht. Ziel dabei ist es, NPs verschiedener Materialien und Größen nebeneinander zu unterscheiden.

Zur schnellen Charakterisierung der NPs sollen leistungsfähige Sensorsysteme zum Einsatz kommen, die die Durchführung der Analysen auch direkt vor Ort ermöglichen. 


\section{Methoden}

Reflektometrische Interferenzspektroskopie

Zum Nachweis der NPs wurde die Reflektometrische Interferenzspektroskopie (RIfS) eingesetzt [5-7]. Diese optische und zugleich markierungsfreie Methode ermöglicht es, Bindungsprozesse zeitaufgelöst zu verfolgen. Die Bindungssignale resultieren dabei aus Wechselwirkungen zwischen Analyten und sensitiver Schicht. Die sensitive Schicht dient hierbei als Erkennungselement und ist somit von zentraler Bedeutung, da eine selektive Wechselwirkung mit den Analyten für einen Nachweis entscheidend ist. In der vorliegenden Arbeit wurde Polycinnamat als sensitive Schicht zum Nachweis von SilberNanopartikeln genutzt.

Die Detektion von Wechselwirkungen erfolgt über einen Glastransducer, der mit einer sensitiven Schicht beschichtet ist und mit Weißlicht bestrahlt wird (s. Fig. 1). Das eingestrahlte Licht wird an verschiedenen Schichten reflektiert bzw. transmittiert (s. Fig. 1), wobei ein charakteristisches Interferenzsmuster entsteht (s. Fig. 2). Sobald die Analyten über einen Transducer geleitet werden und ein Bindungsprozess stattfindet, ändert sich die optische Schichtdicke, die vom Brechungsindex so wie der physikalischen Schichtdicke abhängig ist. Dabei verschiebt sich das charakteristische Interferenzspektrum (s. Fig. 2) und es ergibt sich eine Bindungskurve, die sich aus der zeitabhängigen Verschiebung der Maxima errechnet (s. Fig. 3).

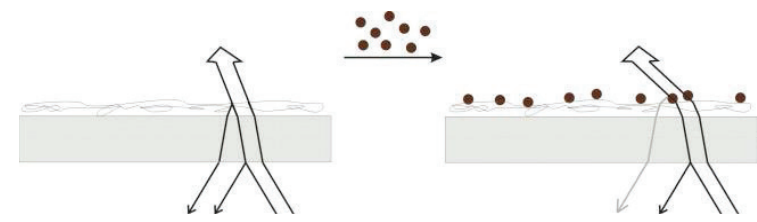

Fig. 1. Wechselwirkung zwischen Analyten und sensitiver Schicht [8].

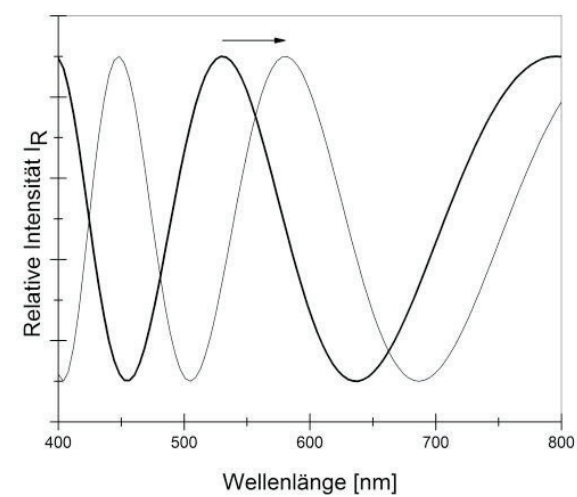

Fig. 2. Verschiebung des Interferenzspektrums [8].

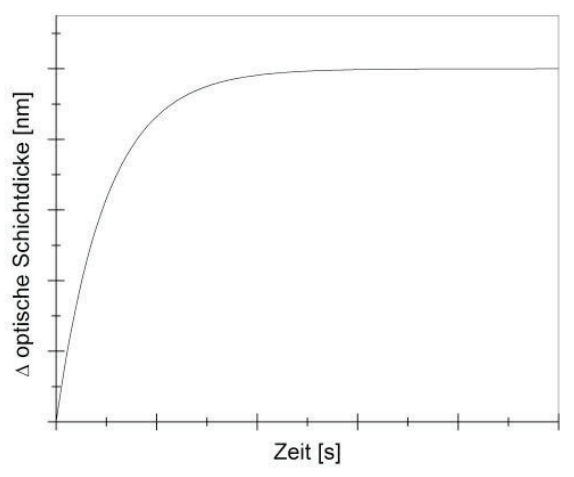

Fig. 3. Resultierende Bindungskurve [8].

Spin-Coating

$\mathrm{Zu}$ Beschichtung der Transducer wurde das Spin-Coating-Verfahren eingesetzt. In Fig. 4 ist das Verfahren schematisch dargestellt. Zunächst wird das Polymer in einem geeigneten Lösungsmittel gelöst und auf den Glastransducer aufgetragen. Im Anschluss wird der Transducer in Rotation versetzt, so dass überschüssiges Lösungsmittel aufgrund von Zentrifugalkräften heruntergeschleudert wird und sich eine dünne Polymerschicht ausbildet [9].
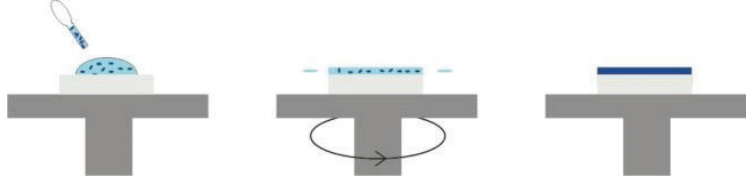

Fig. 4. Beschichtung Verfahren [8].

mittels

Spin-Coating-

\section{Ergebnisse \& Diskussion}

Im Rahmen dieser Arbeit wurden SilberNanopartikel mit zwei unterschiedlichen Größen im zweistelligen Nanometerbereich untersucht. Neben dem Einfluss der Größe, wurde auch untersucht, wie sich verschiedene Lagerungszeiten auf die Messergebnisse auswirken.

Einfluss der Lagerungszeit auf RIfS-Messungen

Nach dem Beschichten der Transducer, wurden diese unterschiedlich lange gelagert, um zu sehen, ob die Oberflächen über einen längeren Zeitraum stabil sind. Dazu wurden zunächst RIfS-Messungen auf Transducern durchgeführt, die einen Tag lang gelagert wurden. Als Analyten dienten Silber-Nanopartikel, die hier als Ag19-Partikel bezeichnet werden. Nach einer Lagerung von zwei Wochen wurden auf weiteren Transducern RIfS-Messungen mit den gleichen Partikeln durchgeführt. In Fig. 5 sind die Änderungen der optischen Schichtdicken während der Messungen auf drei 
verschiedenen Transducern, die einen Tag gelagert wurden, dargestellt. Eine Wechselwirkung der Partikel mit der Oberfläche kann hier ausgeschlossen werden, da zu Beginn (bei 500 s) als auch zum Ende (bei 1600 s) der Probeninjektion ein sprunghafter Anstieg bzw. Abfall der optischen Schichtdicken zu erkennen ist. Die Ursache hierfür ist ein reiner Brechungsindexsprung.

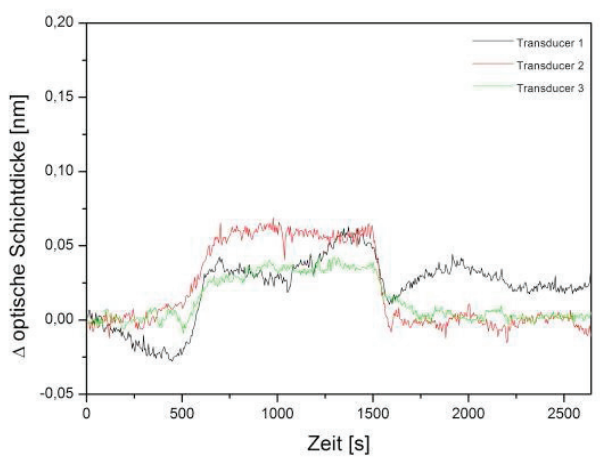

Fig. 5. Bindungskurven von drei verschiedenen Messungen mit Silber-Nanopartikeln Ag19 auf unterschiedlichen Transducern nach einer Lagerung von einem Tag [8].

In Fig. 6 zeigt der ansteigende Verlauf der Bindungskurven deutlich, dass nach einer Lagerung von zwei Wochen eine Wechselwirkung zwischen Silber-Nanopartikeln Ag19 und der sensitiven Schicht stattgefunden hat. Die unterschiedlichen Änderungen der optischen Schichtdicken zwischen 0,2 und 0,7 $\mathrm{nm}$ deuten darauf hin, dass die Transducer nicht homogen beschichtet wurden. Dieses Problem muss in Zukunft gelöst werden, um auch einen quantitativen Nachweis der Partikel zu ermöglichen.

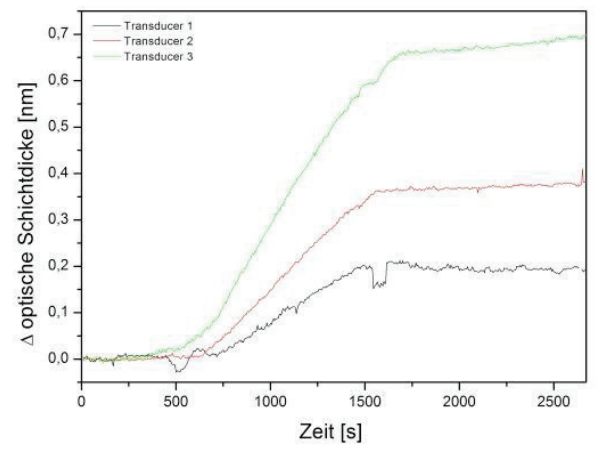

Fig. 6. Bindungskurven von drei verschiedenen Messungen mit Silber-Nanopartikeln Ag19 auf unterschiedlichen Transducern nach einer Lagerung von zwei Wochen [8].

Beim Vergleich von Fig. 5 mit Fig. 6 wird deutlich, dass die Lagerungszeit einen Einfluss auf die Messergebnisse hat. Demzufolge ändert sich die Oberfläche während der Lagerung und sollte so präpariert werden, dass sie über einen längeren Zeitraum stabil ist.

\section{Einfluss der Partikelgröße auf RIfS-Messungen}

Da Nanopartikel in den unterschiedlichsten Größen eingesetzt werden, muss ein Ziel sein, die Nanopartikel nicht nur nach Material, sondern auch nach Größe zu unterscheiden. Im Falle von Silber-Nanopartikeln ist dies mit Hilfe der RIfS für zwei unterschiedliche Größen gelungen.

Neben den Silber-Nanopartikeln Ag19, konnten auch andere Silber-Nanopartikel, als Ag20Partikel bezeichnet, nachgewiesen werden. Die Bindungskurve dieser Partikel ist in Fig. $7 \mathrm{zu}$ sehen. Am Verlauf der Bindungskurve ist $\mathrm{zu}$ erkennen, dass ein Teil der Nanopartikel nach Ende der Probeninjektion (bei 1600 s) wieder von der Oberfläche abgetragen wird. Vergleicht man die Bindungskurven in Fig. 6 und Fig. 7 miteinander, so sind unterschiedliche Signalformen zu erkennen. Ein weiterer Unterschied besteht in der Signalhöhe, so dass verschieden große Nanopartikel, aufgrund ihres unterschiedlichen Bindungsverhaltens gegenüber Polycinnamat, qualitativ detektiert werden können.

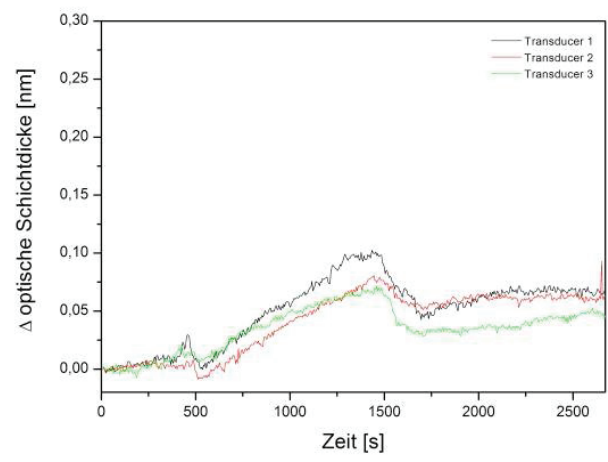

Fig. 7. Bindungskurven von drei verschiedenen Messungen mit Silber-Nanopartikeln Ag20 auf unterschiedlichen Transducern nach einer Lagerung von zwei Wochen [8].

\section{Ausblick}

In Zukunft sollte versucht werden, die Oberflächen so zu stabilisieren, dass auch eine Lagerung über einen längeren Zeitraum keinen Einfluss mehr auf die Messergebnisse hat. Außerdem müssen weitere Partikelgrößen untersucht werden. Wünschenswert ist weitergehend auch ein Nachweis von Nanopartikeln aus anderen Materialien wie z.B. Gold. 


\section{Danksagung}

Die Durchführung dieser Arbeit fand im Rahmen des, von der EU geförderten, Projekts INSTANT (Innovative Sensor for the Analysis of Nanoparticles in Selected Target Products) statt.

Die Nanopartikel wurden von der Bundesanstalt für Materialforschung und -prüfung als Referenzmaterialien zur Verfügung gestellt.

\section{Literatur}

[1] Schulz, J.; et al.: Distribution of sunscreens on skin. In: Advanced Drug Delivery Reviews 54 (2002), S. S157-S163

[2] Silver, S.; Phung L.; Silver G.: Silver as biocides in burn and wound dressings and bacterial resistance to silver compounds. In: Journal of Industrial Microbiology and Biotechnology 33 (2006), Nr.7, S. 627-634

[3] Umweltbundesamt: Beurteilung eines möglichen Krebsrisikos von Nanomaterialien und von aus Produkten freigesetzten Nanopartikel. http://www.umweltbundesamt.de/sites/default/file s/medien/publikation/long/4068.pdf

Stand: 06.07.2015

[4] Grobe, A.,; et al.: Nanotechnologien aus der Sicht von Konsumenten. Was Verbraucher wissen und was sie wissen wollen. Studie i.A. BAG Bundesamt für Gesundheit, Bern \& Stiftung Risiko-Dialog, 2012.

[5] Rau, S.; Hilbig, U.; Gauglitz, G.: Label-free optical biosensor for detection and quantification of the non-steroidal anti-inflammatory drug diclofenac in milk without any sample pretreatment. In: Anal. Bioanal. Chem. 406 (2014), Nr.14, S.3377-3386

[6] Ewald, M.; Le Blanc, A.F.; Gauglitz, G.; Proll, G.: A robust sensor platform for label-free detection of anti-Salmonella antibodies using undiluted animal sera. In: Anal. Bioanal. Chem. 405 (2013), Nr.20, S.6461-6469

[7] Brecht, A.; Gauglitz, G.; Nahm, W.: Interferometric measurements used in chemical and biochemical sensors. In: Analysis 20 (1992), Nr.3, S.135-140

[8] Adam, J.: Polymerbasierte Erkennungselemente zum Nachweis von Nanopartikeln mittels Reflektometrischer Interferenzspektroskopie, Eberhard Karls Universität Tübingen, Diplomarbeit, 2015

[9] Norrman, K.; Ghanbari-Siahkali, A,; Larsen, N. B.: 6 Studies of spin-coated polymer films. In: Annual Reports Section "C" (Physical Chemistry) 101 (2005), S. 174-201 